\title{
Blind Estimation of Spreading Sequence based on the Wide Window and PAST Algorithm
}

\author{
Min Li \\ Institute of Information Engineering, Harbin Institute of \\ Technology \\ Weihai, China \\ limincom@126.com \\ Jinghong Xue \\ Institute of Information Engineering, Harbin Institute of \\ Technology \\ Weihai, China \\ Xuejing_h@126.com
}

\author{
Bin Xu \\ Institute of Information Engineering, Harbin Institute of \\ Technology \\ Weihai , China \\ saturdaybin1118@163.com \\ Mengmeng Wang \\ Institute of Information Engineering, Harbin Institute of \\ Technology \\ Weihai , China \\ ddd1231992@163.com
}

\begin{abstract}
The problem of blind estimation of spreading sequence is an important aspect in the context of spectrum surveillance, which the pseudo-random sequence used by the transmitter is unknown. In this paper, the approach of the spreading sequence recovery is proposed by using projection approximation subspace tracking (PAST) method and sliding window technique, when the received signal is sampled and divided into temporal windows, the size of which is double the pseudorandom sequence period. The calculation of the main eigenvector is only needed, and sliding window technique is used to realize the spreading sequence synchronization. The PAST method avoids the calculation of eigenvalue decomposition, the main way is to obtain the convergence weights in an iterative method. Therefore, it is easy to realize in hardware and has less computational cost. Experimental results show that the proposed method eliminates the problem of phase ambiguity and can provide a good estimation, even when the received signal is far below the noise level.
\end{abstract}

Keywords—spreading sequence; subspace tracking; sliding window; phase ambiguity; blind estimation

\section{INTRODUCTION}

Direct Sequence Spread Spectrum (DSSS) communication has been widely used in secret communication and code division multiple access (CDMA) system in recent decades. Indeed, DSSS transmitters use a periodical pseudo-random sequence to modulate the baseband signal before transmission. With high rate of spreading sequence in the original signal spectrum extension, it has a series of advantages, such as large information transmission capacity, good interference immunity and being hard to be detected. Meanwhile, it can work well in a very low signal-to-noise ratio. However, these characteristics bring difficulties to communication surveillance and electronic countermeasures. Thus the research on detection and estimation of spread spectrum signal is of great significance, and the realization of blind dispreading techniques is an important aspect.
Two types of estimating the spreading sequence without prior knowledge about the transmitter have been mainly considered. One is the eigenanalysis method [2], and the other is neural network method [3-4]. The eigenanalysis method is based on eigenanalysis techniques. The received signal is divided into windows, from which a covariance matrix is computed. The spreading sequence can be reconstructed from the two first eigenvectors of the covariance matrix. The neural network methods use the adaptive tapped delay line FIR filter updated by the constrained Hebbian rule to estimate the spreading sequence.

In [2] and [3], the projection approximation subspace tracking(PAST) algorithm is brought forward, which is a real-time fast algorithm of eigenanalysis method, the weights converge to the main eigenvectors through the process of adaptive iteration. The PAST method avoids the eigenvalue decomposition and reduces the amount of calculation greatly. But there is no solution in the phase ambiguity problem in the process of vector joining together.

In [3], wide window method is applied to the eigenanalysis method is proposed to solve the problem of phase ambiguity caused by the vector stitching. However, the received signal is divided by wide windows, making the computational amount is increased and can't realize the real-time blind estimation. Meanwhile, it is infeasible in the actual situation, because of the big storage space requirements for hardware.

In this paper, we combine the PAST method and the wide windows together in order to further reduce the amount of calculation. This method avoids the phase ambiguity, and can estimate the asynchronous value at the same time, for which the received signal is sampled and divided into temporal windows, the size of which is double the pseudorandom sequence period. Experimental results show that this algorithm eliminates the problem of phase ambiguity and can provide a good estimation, even when the received signal is far below the noise level. 
The paper is organized as follows. In section II , we give the notations and hypotheses and the proposed approach is described. Finally, numerical results are provided to illustrate the method in section III and a conclusion is stated in section IV.

\section{NOTATIONS AND HYPOTHESES}

\section{A. Eigenanalysis algorithm}

In a DSSS transmission, the symbols $a_{k}$ are multiplied by a pseudo-random sequence $\left\{c_{k}, k=1,2, \cdots, P\right\}$ of chip duration $T_{c}$, which spreads the bandwidth.

The spread signal $s(t)$ must be modeled as

$$
s(t)=c(t) d(t)(1)
$$

Where, $c(t)$ is the spreading sequence, $d(t)$ is the message signal and $s(t)$ is the DSSS baseband signal at the output of the receiver filter.

The received demodulated baseband signal $y(t)$ in the general single user environment can be written as:

$$
y(t)=s(t)+n(t)(2)
$$

Where $n(t)$ is the noise at the output of the receiver filter and is uncorrelated with the signal.

$$
\begin{aligned}
& s(t)=\sum_{k=-\infty}^{+\infty} a_{i} c(t-i T) \\
& c(t)=\sum_{k=0}^{P-1} c_{k} p\left(t-k T_{c}\right)
\end{aligned}
$$

Where, $p(t)$ is the convolution of the transmission filter, the channel filter (which represents the channel echoes) and the receiver filter; $\left\{c_{k}, k=1,2, \cdots, P\right\}$ is the pseudo-random sequence; $P$ is the length (number of bits) of the pseudo-random sequence. $T$ is the symbol period; $T_{c}$ is the chip period $\left(T_{c}=T / P\right)$.

In the context of spectrum surveillance, the pseudorandom sequence used by the transmitter is unknown (as well as other transmitter parameters such as duration of the sequence, symbol frequency and carrier frequency). In the paper, we assume that the symbol period $T$ has been estimated and all other parameters are known. The sampling period can be set to $T_{s}=T_{c}$. Eigenanalysis method is based on eigenanalysis techniques. The received signal is sampled and divided into non-overlapping temporal windows, the duration of which is $T$, from which a covariance matrix is computed.

Let us note $\boldsymbol{y}$ the content of a window and define the correlation matrix $\boldsymbol{R}=E\left[\boldsymbol{y} \cdot \boldsymbol{y}^{\mathrm{H}}\right]$, where ${ }^{\mathrm{H}}$ denotes the Hermitian transpose. The non-negative eigenvalues and the corresponding orthonormal eigenvectors of matrix $\boldsymbol{R}$ are denoted by $\lambda_{i}$ and $\mu_{i}(\mathrm{i}=1,2, \ldots, P)$. It has been proved that the eigenanalysis of this matrix yields two large eigenvalues:

$$
\lambda_{1}=\left(1+\rho \frac{T-t_{0}}{T_{e}}\right) \sigma_{n}^{2}, \quad \lambda_{2}=\left(1+\rho \frac{t_{0}}{T_{e}}\right) \sigma_{n}^{2}
$$

It is shown that the spreading sequence can be reconstructed from the two first eigenvectors of this matrix, and some useful information, such as the desynchronization time, can be extracted from the eigenvalues. However, the desynchronization time $\hat{t_{0}}$ must be estimated to know which eigenvector describes the beginning of the sequence and which one the end. The signal to noise ratio (SNR) $\hat{\rho}$ and the desynchronization $\hat{t_{0}}$ can be estimated can be extracted from the eigenvalues.

$$
\hat{\rho}=\left(\frac{\lambda_{1}+\lambda_{2}}{\sigma_{n}^{2}}-2\right) \frac{T_{e}}{T}, \hat{t_{0}}=\frac{T_{e}}{\hat{\rho}}\left(\frac{\lambda_{2}}{\sigma_{n}^{2}}-1\right) \text { (6) }
$$

It is obvious that the less accurate the value of $\hat{t_{0}}$ will be, the higher the noise variance $\sigma_{n}^{2}$ will be.

As a result of the received signal covariance function is positive definite matrix, two large eigenvectors converges to spread spectrum code or negative spread spectrum code instead, there exists phase ambiguity, unable to complete the joining together of spreading sequences better.

\section{B. PAST algorithm}

The In [2], projection approximation subspace tracking algorithm (PAST) was put forward by Yang in 1995. It relies on a novel interpretation of the signal subspace as the solution of an unconstrained minimization problem. The main way is to solve the convergence weights in an iterative method, is also the process of computing signal subspace. Let $\boldsymbol{x}$ be a complex valued random vector process with the correlation matrix $\boldsymbol{R}=\mathrm{E}\left[\boldsymbol{x} \boldsymbol{x}^{\mathrm{H}}\right]$. Consider the following scalar function

$$
\begin{aligned}
J(\boldsymbol{W}) & =E\left\|\boldsymbol{x}-\boldsymbol{W} \boldsymbol{W}^{\mathrm{H}} \boldsymbol{x}\right\|^{2} \\
& =\operatorname{Tr}[\boldsymbol{R}]-2 \operatorname{Tr}\left[\boldsymbol{W}^{H} \boldsymbol{R} \boldsymbol{W}\right]+\operatorname{Tr}\left[\boldsymbol{W}^{H} \boldsymbol{R} \boldsymbol{W} \boldsymbol{W}^{H} \boldsymbol{W}\right]
\end{aligned}
$$

Where $\operatorname{Tr}[\cdot]$ denotes the matrix trace operation, $\boldsymbol{x}$ and $\boldsymbol{W}$ are the received signal vector and weight vector respectively.

$\boldsymbol{W}$ is a stationary point of $J(\boldsymbol{W})$ if and only if $\boldsymbol{W}=\boldsymbol{U}_{M} \boldsymbol{Q}$ where $\boldsymbol{U}$ contains any $M$ distinct eigenvectors of $\boldsymbol{R}$ and $\boldsymbol{Q}$ is an arbitrary unitary matrix. At each stationary point, $J(\boldsymbol{W})$ equals the sum of eigenvalues whose eigenvectors are not involved in $\boldsymbol{U}_{M}$.

All stationary points of $J(\boldsymbol{W})$ are saddle points except when $\boldsymbol{U}_{M}$ contains the $M$ dominant eigenvectors of $\boldsymbol{R}$. In this case, $J(\boldsymbol{W})$ attains the global minimum. In addition, 
$\boldsymbol{W} \boldsymbol{W}^{H}$ is unique, denotes the projection matrix of signal subspace. Since $J(\boldsymbol{W})$ has a global minimum at which the column span of $\boldsymbol{W}$ equals the signal subspace and no other local minima, a global convergence is guaranteed if one seeks the signal subspace of $\boldsymbol{C}$ by minimizing $J(\boldsymbol{W})$ via iterative methods.

The so called projection approximation subspace tracking (PAST) approach. Replacing the expectation in (8) with the exponentially weighted sum yields

$$
\begin{aligned}
J(\boldsymbol{W}(t)) & =\sum_{i=1}^{t} \beta^{t-i}\left\|\boldsymbol{x}(i)-\boldsymbol{W}(t) \boldsymbol{W}^{\mathrm{H}}(t) \boldsymbol{x}(i)\right\|^{2} \\
& =\operatorname{Tr}[\boldsymbol{R}(t)]-2 \operatorname{Tr}\left[\boldsymbol{W}^{H}(t) \boldsymbol{R}(t) \boldsymbol{W}(t)\right] \\
& +\operatorname{Tr}\left[\boldsymbol{W}(t)^{H} \boldsymbol{R}(t) \boldsymbol{W}(t) \boldsymbol{W}^{H}(t) \boldsymbol{W}(t)\right]
\end{aligned}
$$

All sample vectors available in the time interval $1<i<t$ are involved in estimating the signal subspace at the time instant $t$. The use of the forgetting factor $0<\beta<1$ is intended to ensure that data in the distant past are downweighted in order to afford the tracking capability when the system operates in a nonstationary environment.

$$
J(\boldsymbol{W}(t)) \text { in (8) is obviously identical to } J(\boldsymbol{W}) \text { in (7) }
$$
except for the use of the exponentially weighted sample correlation matrix

$$
\boldsymbol{R}(t)=\sum_{i=1}^{t} \beta^{t-i} \boldsymbol{x}(i) \boldsymbol{x}^{\mathrm{H}}(i)=\beta \boldsymbol{R}(t-1) \boldsymbol{x}(t) \boldsymbol{x}^{\mathrm{H}}(t)
$$

instead of $\boldsymbol{R}=\mathrm{E}\left[\boldsymbol{x} \boldsymbol{x}^{\mathrm{H}}\right]$.

$J(\boldsymbol{W}(t))$ is a fourth-order function of the elements of $\boldsymbol{W}(t)$. Iterative algorithms are thus necessary to minimize $\boldsymbol{W}^{\mathrm{H}}(t) \boldsymbol{x}(i)$. The key issue of the PAST approach is to approximate (8), the unknown projection of $\boldsymbol{x}(i)$ on to the columns of $\boldsymbol{W}(t)$ by the expression $y(i)=\boldsymbol{W}^{\mathrm{H}}(i-1) \boldsymbol{x}(i)$.

This results in another expression for $J(\boldsymbol{W})$ is as follows:

$$
J^{\prime}(\boldsymbol{W}(t))=\sum_{i=1}^{t} \beta^{t-i}\|\boldsymbol{x}(i)-\boldsymbol{W}(t) y(i)\|^{2}
$$

which is quadratic in the elements of $\boldsymbol{W}(t)$.

Recursive least squares (RLS) methods can then be applied to track the signal subspace efficiently, then RLS criterion is applied to obtain convergence weights by iterative operation, and the subspace decomposition can be converted to subspace iteration, the acquired convergence weights are the main eigenvector of received signal covariance matrix.

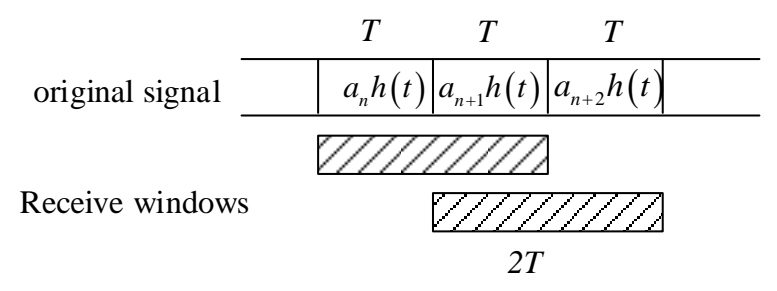

Figure 1.The division of received signal based on the wide window

\section{PAST algorithm based on wide windows}

In this paper, the wide window method and the PAST method are combined, in this case, the wide window is twice that of spreading sequence cycle is $2 T$, and adjacent overlapping width of the window to $T$, the concrete implementation process is shown in figure 1 .

The figure 1 shows each received the window contains a complete cycle of spread spectrum code sequence, then use PAST algorithm to quickly close to main eigenvector of the matrix related to the reception In order to further improve the convergence performance, the introduction of forgetting factor and weight adjustment vector, the projection approximation subspace tracking algorithm steps are as follows:

a) The received signal is sampled at $T_{c}$ and divided as figure 1 , the sampled signal can be written as $x_{k}(n), k=1,2, \cdots, Q ; n=1,2, \cdots, 2 P$.Where, $x_{k}(n)$ stands for the received signal of the $k$ th window, $Q$ is the number of windows, $2 P$ is the dimension of the signal.

b) For $i=0$, choose $\boldsymbol{w}_{i}(0)$ and $g(0)$ suitably, we can set $g(0)=0.01, \omega_{0}(0)=[1,0,0, \ldots, 0]^{T}$

c) For $i=1,2, \cdots, Q$, do iteration as follows:

$$
\begin{gathered}
y(i)=\boldsymbol{w}_{i}^{H} \boldsymbol{x}_{i}(11) \\
h(i)=g(i) * y(i)(12) \\
k(i)=h(i) /[\beta+y(i) * h(i)](13) \\
\boldsymbol{e}_{i}=\boldsymbol{x}_{i}-y(i) \boldsymbol{w}_{i-1}(14) \\
\boldsymbol{w}_{i}=\boldsymbol{w}_{i-1}+\left[\boldsymbol{x}_{i}-y(i) * \boldsymbol{e}_{i}\right] / k(i)(15) \\
g(i)=[g(i-1)-k(i) * y(i) * g(i-1)] / \beta
\end{gathered}
$$

Convergence weights of the spread spectrum code can be obtained through the above steps, there is no need to get all the signal vectors, only need to extract the main eigenvectors. To estimate the time delay by sliding window method, can get the estimate of spread spectrum code sequence by cutting the main eigenvector.

Known from the analysis of the above, through the PAST method to extract the primary feature vector, we will combine sliding window method to estimate the asynchronous time delay. First, let us 
note $\hat{\mathbf{V}}=\left[v_{1}, v_{2}, \cdots, v_{2 P-1}\right]$ the principal eigenvector. We define a scalar function:

$$
J\left(\hat{\boldsymbol{V}}_{k}\right)=\left\|\hat{\boldsymbol{V}}_{k}\right\|_{2}^{2}=v_{k}^{2}+v_{k+1}^{2}+\cdots+v_{k+P-1}^{2} \quad k=1,2, \cdots, P
$$

Calculate for the square of 2-norm lengths of $P$ elements in a sliding window.

Sliding window in figure 2 , each shift a code width, the definition of scalar function $J\left(\hat{\boldsymbol{V}}_{k}\right)$ obtain the maximum value, when a sliding window of the principal eigenvector is signal vector, $k$ value at this time is denoted by $k_{\max }$. According $k_{\max }$ to intercept vector, we get the estimated spreading sequence as shown in formula (18)

$$
\hat{\boldsymbol{C}}=\operatorname{sgn}\left(\hat{\boldsymbol{V}}_{k_{\max }}\right)=\operatorname{sgn}\left(\left[v_{k_{\max }}, v_{k_{\max }+1}, \cdots, v_{k_{\max }+P-1}\right]\right)
$$

Sliding window method is shown in figure 2.
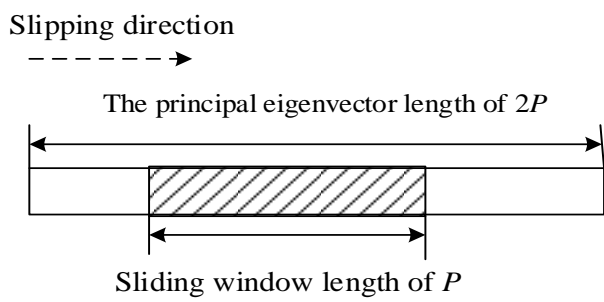

Figure 2..The design of the sliding window

In this paper, we set desynchronization $t_{0}=k_{0} T_{c}$, for the estimation of the synchronization points $k_{0}$ is calculated as shown in formula (19)

$$
\begin{cases}k_{\max }<N / 2, & k_{0}=k_{\max }+\frac{P-1}{2} \\ k_{\max } \geq N / 2, & k_{0}=k_{\text {max }}-\frac{P+1}{2}\end{cases}
$$

$k_{\max }$ is obtained when scalar function $J\left(\hat{\mathbf{V}}_{k}\right)$ gets the maximum value.

\section{EXPERIMENTAL SIMULATIONS}

\section{A. Experimental results}

To illustrate the performance of the method proposed in this paper, a DS-SS signal is generated using a randomsequence of length $P=127$. We adopt the balance GOLD sequences as spreading sequences, which are traditionally used in CDMA systems. The symbols belong to a Binary Phase Shift Keying (BPSK) constellation. The SNR is $9 \mathrm{~dB}$, hence, the noise power in the signal passband is considerably larger than the signal power. 200 windows are used for estimating the weights of PAST method. To simplify the interpretation of illustrations, the sampling period is chosen equal to $T_{s}=T_{c}$. Set the desynchronization time $t_{0}=40 T_{c}$.The principal eigenvector and the estimated desynchronization time $\hat{t}_{0}$ are shown in figure 3 .

Figure 3 shows the results of the estimated principal eigenvector and desynchronization time. It is obvious that the length of the estimated principal eigenvector is $2 P$. We can cut the estimated principal eigenvector according to the desynchronization time. Formula (19) gives the calculating method of $k_{\max }$, thus, we can get $k_{\max }=40$, eliminating the phase ambiguity. The experimental simulations verify the effectiveness of the proposed method.
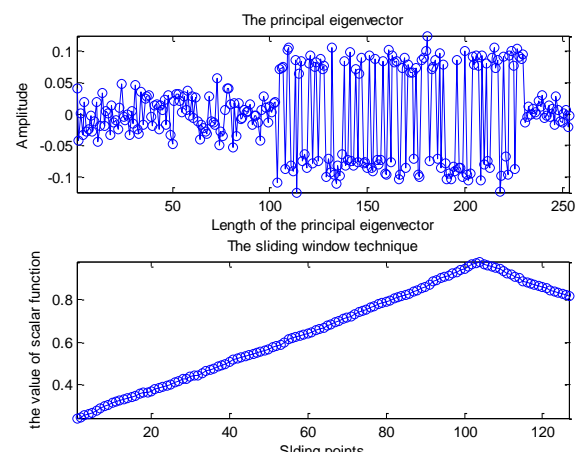

Figure 3. The principal eigenvector and the estimated desynchronization time

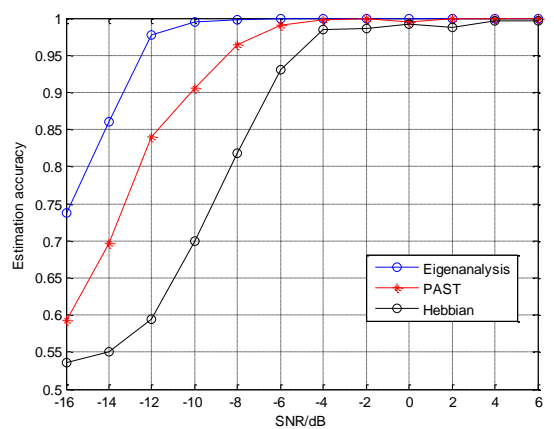

Figure 4. The performance of PN codes estimates based on wide window method

Figure 4 shows the performance of PAST method based on wide window method. The proposed method can provide a good estimation in low SNR. The estimation accuracy can get 100 percent in $-6 \mathrm{~dB}$ and the performance is superior to the neural network method based on Hebbian rule.

\section{B. Analysis of the calculation amount}

We theoretically analyzed the calculation of the proposed method, the number of received signal data is $Q$ groups. The computation of eigenanalysis method is $2 P \cdot 2 P \cdot Q=4 P^{2} Q$

As for the proposed method in this paper, the computation is $2 P Q$, ignoring the addition and subtraction of vectors.

With the increase of received data groups $Q$, the calculation amount of eigenanalysis method increase greatly. Considering the actual situation, to further improve the estimation performance, more data have to be dealt with, eigenanalysis method lose the real-time signal 
processing Therefore, the proposed algorithm can be applied to real-time blind expansion solution system.

\section{CONCLUSION}

In this paper, in order to avoid the phase ambiguity problem caused by the vector splicing, the wide window method is applied to the projection approximation subspace tracking algorithm, only need to extract the principal eigenvector. Meanwhile the principal eigenvector scalar function is defined in this paper and the sliding window technique is used to achieve the principal eigenvector cutting. Experimental results show that the proposed method still can restore the spreading sequences at low SNR similar to the traditional eigenanalysis method, but the amount of calculation is reduced a lot. The method has good real-time performance and is easier to realize in the hardware.

\section{REFERENCES}

[1] Burel G, Bouder C. Blind estimation of the pseudo-random sequence of a direct sequence spread spectrum signal[C]. //MILCOM 2000. 21st Century Military Communications Conference Proceedings. IEEE, 2000:967 - 970.

[2] Yang Bin. An extension of the PASTd algorithm to both rank and subspace tracking[J]. IEEE Signal Processing Letters, 1995, 2(9) : 179-182.

[3] Yang B. Convergence analysis of the subspace tracking algorithms PAST and PASTd[C]. //Acoustics, Speech, and Signal Processing, Icassp-96, Conference Proceedings, IEEE International Conference on. IEEE, 1996:1759 - 1762 .

[4] Ma chao, Li-min Zhang, Zhao-gen Zhong etc. Based on the double PN spread spectrum sequence periodic narrowband interference blind estimation [J]. Ordnance Industry Automation, 2013, (12).

[5] Cheng-cheng Li, Fang-zong Wang. Based on projection approximation subspace tracking algorithm of harmonic detection method $[\mathrm{J}]$. Electric measurement and instrument, 2009, 46 (4). The DOI: 10.3969 / j.i SSN. 1001-1390.2009.04.006.

[6] Hong-bo zhang, Lv Ming. Spread spectrum code blind estimation algorithm based on subspace tracking $[\mathrm{J}]$. Journal of systems engineering and electronics, 2006, (10). 